\title{
ON NONLINEAR VARIATIONAL INEQUALITIES
}

\author{
MUHAMMED ASLAM NOOR \\ Department of Mathematics \\ College of Science \\ King Saud University, Riyadh 11451, Saudi Arabia \\ (Received June 6, 1990 and in revised form October 20, 1990)
}

\begin{abstract}
The fixed point technique is used to prove the existence of a solution for a class of nonlinear variational inequalities related with odd order constrained boundary value problems and to suggest an iterative algorithm to compute the approximate solution.
\end{abstract}

KEY WORDS AND PHRASES. Variational inequalities, fixed point, odd order boundary problems.

1980 AMS (MOS) SUBJECT CLASSIFICATION CODES. 49A29, 49A10, 90C30.

\section{INTRODUCTION.}

In recent years, variational inequality theory has been extended and generalized in various directions to study a wide class of problems arising in different branches of mathematical, physical and engineering sciences. We have noted that the theory so far developed is applicable only for studying moving and free boundary values problems of even order. Inspired and motivated by the applications of variational inequality theory, in differential equations and related areas, Noor $[1,2]$ has studied certain classes of variational inequalities related with odd order boundary problems. In this paper, we prove the existence of a solution for a class of nonlinear variational inequalities related with mildly nonlinear constrained elliptic problems using the fixed point technique of Glowinski, Lions and Tremolieres [3], and Noor [4]. This approach enables us to suggest and analyze a general algorithm for these variational inequalities. Several special cases are also discussed.

\section{FORMULATIONS.}

Let $\mathrm{H}$ be a real Hilbert space on which the inner product and norm are denoted by $\left.<_{., \text {.. }}\right\rangle$ and $\|\cdot\|$ respectively. Let $\mathrm{K}$ be a closed nonempty convex set in $\mathrm{H}$.

Given continuous operators $T, A, g: H \rightarrow H$, we consider the problem of finding $u \varepsilon H$ such that $g(u) \varepsilon K$ and 


$$
\langle T u, g(v)-g(u)>\geq\langle A(u), g(v)-g(u)>\text {. for all } g(v) \varepsilon K
$$

The inequalities of the type (2.1) are known as nonlinear variational inequalities. For physical formulation and iterative methods, see Noor [2].

SPECIAL CASES.

(i) If $\mathrm{A}(\mathrm{u}) \equiv 0$, then problem (2.1) reduces to finding $u \varepsilon K$ such that $g(u) \varepsilon K$ and

$$
<T u, g(v)-g(u)>\geq 0 \quad \text { for all } g(v) \varepsilon K
$$

which has been introduced and studied by Noor [1].

(ii) If $K^{*}$ is the polar cone of the convex cone in $\mathrm{H}$, then problem (2.1) is equivalent to finding $u \varepsilon H$ such that

$$
g(u) \varepsilon K, \quad(T u-A(u)) \varepsilon K^{*} \text { and }<T u-A(u), g(u)>=0
$$

This is known as the generalized nonlinear complementarity problem and appears to be new one.

(iii) If $g \equiv I$, the identity operator, then problem (2.1) is equivalent to finding $u \varepsilon K$ such that

$$
\langle T u, v-u\rangle \geq\langle A(u), v-u\rangle, \text { for all } v \varepsilon K
$$

which is known as the strongly nonlinear variational inequality problem, see Noor [5].

(iv) If $\mathrm{K}=\mathrm{H}$, then problem (2.1) reduces to finding $u \varepsilon H$ such that

$$
<T u-A(u), g(v)>=0, \text { for all } g(v) \varepsilon H,
$$

which is known as the weak formulation of the odd order boundary value problems, see Ciarlet, Schultz and Varga [6].

It is clear that the problem (2.1) is more general and unifying one and includes as special cases previously known many problems.

We also need the following concepts:

DEFINITION 2.1. An operator $\mathrm{T}: \mathrm{H} \rightarrow \mathrm{H}$ is said to be:

(a) Strongly monotone, if there exists a constant $\alpha>0$ such that

$$
<T u-T v, u-v>\geq \alpha\|u-v\|^{2}, \quad \text { for all } u, v \varepsilon H
$$

(b) Lipschitz continuous, if there exists a constant $\beta>0$ such that

$$
\|T u-T v\|<\beta\|u-v\|, \text { for all } u, v \varepsilon H \text {. }
$$

It is obvious that $\alpha<\beta$.

\section{MAIN RESULT.}

We now state and prove the main result of this paper. 
THEOREM 3.1. Let the operators, $\mathrm{T}, \mathrm{g}: \mathrm{H} \rightarrow \mathrm{H}$ be both strongly monotone and Lipschitz continuous. If the operator $\mathrm{A}$ is Lipschitz continuous, then there exists a solution $u \varepsilon H$ such that (2.1) holds.

PROOF. We use the fixed point technique of Glowinski, Lions and Tremolieres [3], and Noor [4] to prove the existence of a solution of (2.1). For given $u \varepsilon H$, we consider the auxiliary problem of finding $w \varepsilon H$, see [3], satisfying the variational inequality

$$
\langle w, v-w\rangle \geq\langle u, v-w>-\rho<T u, g(v)-g(w)>+\rho<A(u), g(v)-g(w)>,
$$

for all $g(v) \varepsilon K, v \varepsilon H$ and some positive constant $\rho$.

Let $w_{1}, w_{2}$ be two solutions of (3.1) related to $u_{1}, u_{2} \varepsilon H$ respectively. It is enough to show that the mapping $u \rightarrow w$ has a fixed point belonging to $H$ satisfying (2.1). In other words, we have to show that for $\rho$ well chosen

$$
\left\|w_{1}-w_{2}\right\| \leq \theta\left\|u_{1}-u_{2}\right\|
$$

with $0<\theta<1$, where $\theta$ is independent of $u_{1}$ and $u_{2}$. Taking $v=w_{2}$ (respectively $v=w_{1}$ ) in (3.1) related to $u_{1}$ (respectively $u_{2}$ ), we have

$$
<w_{1}, w_{2}-w_{1}>\geq<u_{1}, w_{2}-w_{1}>-\rho<T u_{1}, g\left(w_{2}\right)-g\left(w_{1}\right)>+\rho<A\left(u_{1}\right), g\left(w_{2}\right)-g\left(w_{1}\right)>
$$

and

$$
<{ }_{2}, w_{1}-w_{2}>\geq<u_{2}, w_{1}-w_{2}>-\rho<T u_{2}, g\left(w_{1}\right)-g\left(w_{2}\right)>+\rho<A\left(u_{2}\right), g\left(w_{1}\right)-g\left(w_{2}\right)>
$$

Adding these inequalities, we have

$$
\begin{aligned}
<w_{1}-w_{2}, w_{1}-w_{2}>\leq & <u_{1}-u_{2}, w_{1}-w_{2}>-\rho<T u_{1}-T u_{2}, g\left(w_{1}\right)-g\left(w_{2}\right)> \\
& +\rho<A\left(u_{1}\right)-A\left(u_{2}\right), g\left(w_{1}\right)-g\left(w_{2}\right)>, \\
& =<u_{1}-u_{2}-\rho\left(T u_{1}-T u_{2}\right), w_{1}-w_{2}>+\rho<T u_{1}-T u_{2}, w_{1}-w_{2}-\left(g\left(w_{1}\right)-g\left(w_{2}\right)\right)> \\
& +\rho<A\left(u_{1}\right)-A\left(u_{2}\right), g\left(w_{1}\right)-g\left(w_{2}\right)>,
\end{aligned}
$$

from which it follows that

$$
\begin{gathered}
\left\|w_{1}-w_{2}\right\|^{2} \leq\left\|u_{1}-u_{2}-\rho\left(T u_{1}-T u_{2}\right)\right\|\left\|w_{1}-w_{2}\right\|+\rho\left\|w_{1}-w_{2}-\left(g\left(w_{1}\right)-g\left(w_{2}\right)\right)\right\|\left\|T u_{1}-T u_{2}\right\| \\
+\rho\left\|A\left(u_{1}\right)-A\left(u_{2}\right)\right\|\left\|g\left(w_{1}\right)-g\left(w_{2}\right)\right\|
\end{gathered}
$$

Since $\mathrm{T}, \mathrm{g}$ are both strongly monotone and Lipschitz continuous, so by using the technique of Noor [7], we have

and

$$
\left\|u_{1}-u_{2}-\rho\left(T u_{1}-T u_{2}\right)\right\|^{2} \leq\left(1-2 \alpha \rho+\beta^{2} \rho^{2}\right)\left\|u_{1}-u_{2}\right\|^{2}
$$




$$
\left\|w_{1}-w_{2}-\left(g\left(w_{1}\right)-g\left(w_{2}\right)\right)\right\|^{2} \leq\left(1-2 \sigma+\delta^{2}\right)\left\|\left(w_{1}-w_{2}\right)\right\|^{2}
$$

From (3.2), (3.3) and (3.4), we obtain by using the Lipschitz continuity of $\mathrm{T}, \mathrm{g}$ and $\mathrm{A}$,

$$
\begin{aligned}
\left\|w_{1}-w_{2}\right\| & \leq\left\{\sqrt{1-2 \alpha \rho+\beta^{2} \rho^{2}}+\rho \beta\left(\sqrt{1-2 \sigma+\delta^{2}}\right)+\rho \gamma \delta\right\}\left\|u_{1}-u_{2}\right\| \\
& =\{t(\rho)+\rho(k+\gamma \delta)\}\left\|u_{1}-u_{2}\right\|=\theta\left\|u_{1}-u_{2}\right\|,
\end{aligned}
$$

where $k=\beta\left(\sqrt{1-2 \sigma+\delta^{2}}\right), \quad t(\rho)=\sqrt{ }\left(\overline{1-2 \alpha \rho+\beta^{2} \rho^{2}}\right.$ and $\theta=t(\rho)+\rho(k+\gamma \delta)$.

We have to show that $\theta<1$. Now $t(\rho)$ assumes its minimum value for $\bar{\rho}=\frac{\alpha}{\beta^{2}}$ with $\bar{\rho}=\sqrt{1-\frac{\alpha^{2}}{\beta^{2}}}$.

For $\rho=\bar{\rho}, \rho,(k+\gamma \delta)+t(\rho)<1$ implies that $p<\frac{1}{k+\gamma \delta}$. Thus it follows that $\theta<1$ for all $\rho$ with

$\rho<2 \frac{\alpha-(k+\gamma \delta)}{\beta^{2}-(k+\gamma \delta)^{2}}, \rho<\frac{1}{k+\gamma \delta}$ and $\gamma \delta<\alpha-k$. Since $\theta<1$, so the mapping $u \rightarrow w$ defined by (3.1) has a

fixed point, which is the solution of (2.1), the required results.

REMARK 3.1. We note that the solution of the variational inequality problem (3.1) is equivalent to find the minimum of the auxiliary convex quadratic functional $F(w)$ on $K$ in $H$, where

$$
F(w)=1 / 2<w, w>+\rho<T u-A(u), g(w)>-\langle u, w>
$$

Here $\rho>0$ is a constant. This formulation enables us to suggest an iterative algorithm for finding the approximate solution of the variational inequality problem (2.1).

GENERAL ALGORITHM 3.1. Given the initial value $w_{0}$, solve the problem (3.5) with $u=w_{n}$. If $\left\|w_{n+1}-w_{n}\right\| \leq \varepsilon$, for given $\varepsilon>0$, stop. Otherwise repeat the process with $n=n+2$ and so on.

We point out that general algorithm 3.1 is an innovative and novel way of computing the solution of (2.1) as long as (3.5) is easier to solve than (2.1). Since the problem (3.5) is essentially a minimization problem, so a large number of techniques are available to solve it.

\section{REFERENCES}

1. NOOR, M. ASLAM, General Variational Equalities, Appl. Math. Lett. 1 (1988), 119-122.

2. NOOR, M. ASLAM, An Iterative Algorithm for Variational Inequalities, J. Math. Anal. Appl. to appear.

3. GLOWINSKI, R., LIONS, J. and TREMOLIERES, R. Numerical Analysis of Variational Inequalities, North-Holland, Amsterdam, 1982.

4. NOOR, M. ASLAM, General Nonlinear Variational Inequalities, J. Math. Anal. Appl. 126 (1987), 78-84.

5. NOOR, M. ASLAM, Strongly Nonlinear Variational Inequalities, C.R. Math. Rep. Acad. Sci. Canada, 4 (1982), 213-218.

6. CIARLET, P.G., SCHULTZ, M.H. and VARGA, R.S. Numerical Methods of High Order Accuracy for Nonlinear Boundary Value Problems, V. Monotone Operator Theory, Numer. Math. 13 (1969), 51-77.

7. NOOR, M. ASLAM, An Iterative Scheme for a Class of Quasi Variational Inequalities, $\mathbf{J}$. Math. Anal. Appl. 110 (1985), 463-468. 


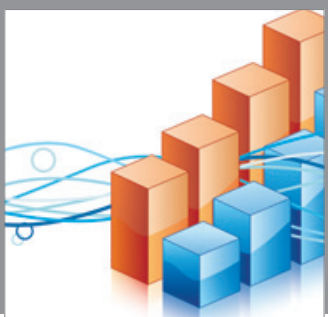

Advances in

Operations Research

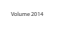

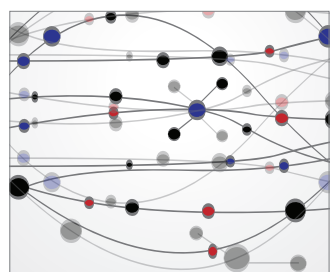

\section{The Scientific} World Journal
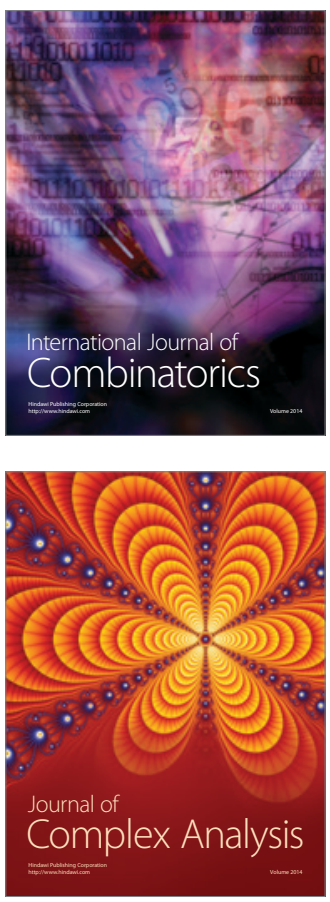

International Journal of

Mathematics and

Mathematical

Sciences
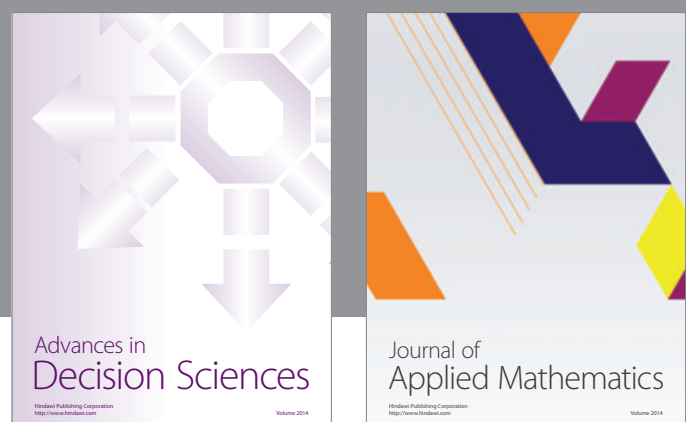

Journal of

Applied Mathematics
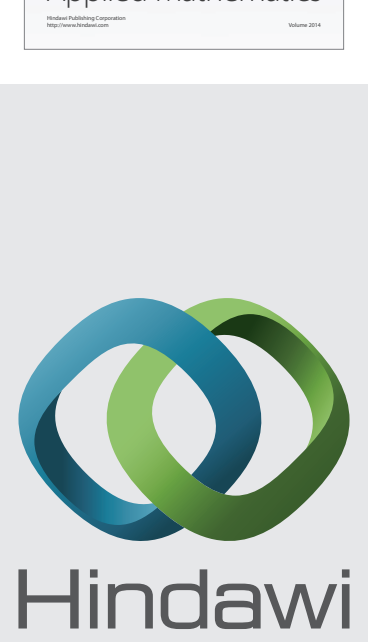

Submit your manuscripts at http://www.hindawi.com
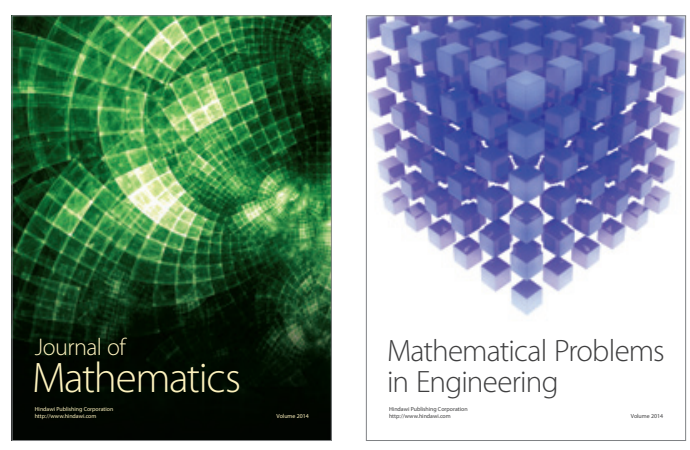

Mathematical Problems in Engineering
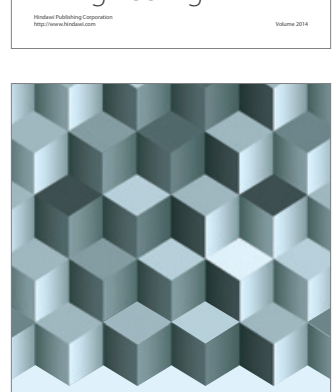

Journal of

Function Spaces
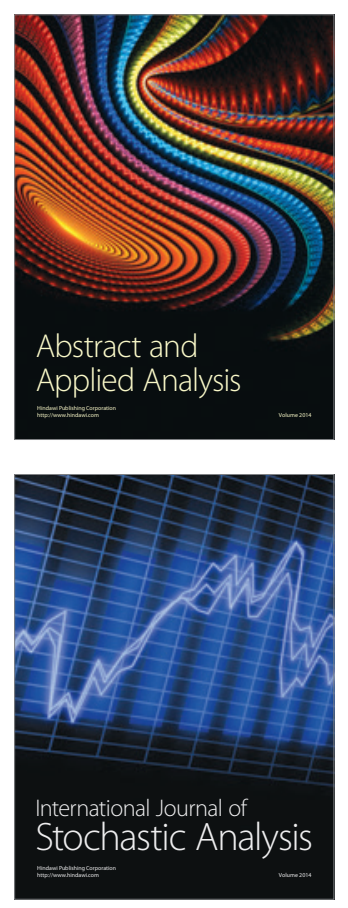

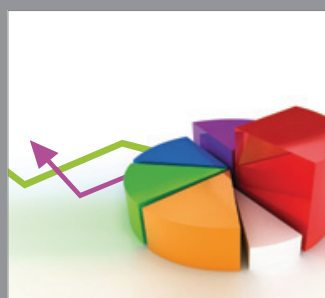

ournal of

Probability and Statistics

Promensencen
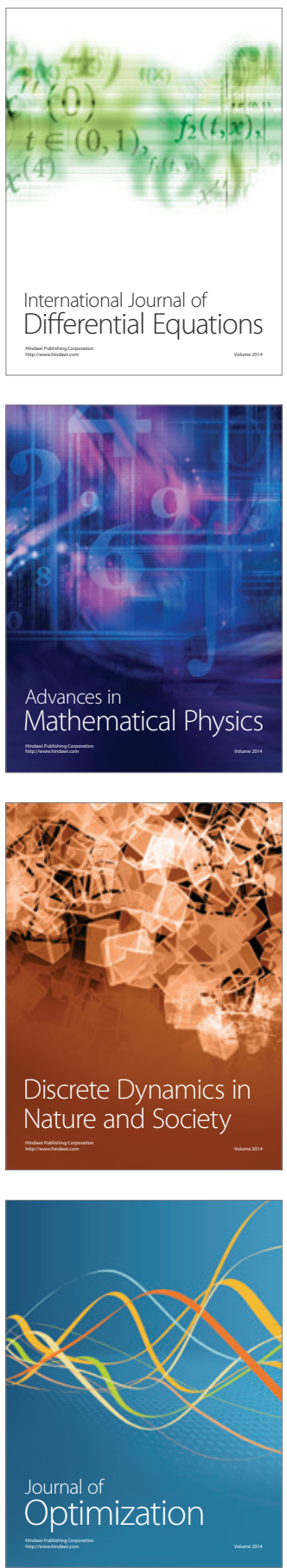